\title{
Hypatulins A and B, Meroterpenes from Hypericum patulum
}

Naonobu Tanaka, ${ }^{*, \dagger}{ }^{\dagger}$ Yuki Yano, ${ }^{\dagger}$ Yutaka Tatano, ${ }^{\S}$ and Yoshiki Kashiwada ${ }^{*, \dagger}$

${ }^{\dagger}$ Graduate School of Pharmaceutical Sciences, Tokushima University, Tokushima 770-8505, Japan,

Graduate School of Bioscience and Bioindustry, Tokushima University, Tokushima 770-8513, Japan,

${ }^{\S}$ Faculty of Pharmaceutical Sciences, International University of Health and Welfare, Ohtawara 324-8501, Japan

\section{Supporting Information}

Experimental Section

Figure S1. $\quad{ }^{1} \mathrm{H}$ NMR spectrum of hypatulin $\mathrm{A}(\mathbf{1})$ in $\mathrm{CD}_{3} \mathrm{OD}(500 \mathrm{MHz})$.

Figure S2. $\quad{ }^{13} \mathrm{C}$ NMR spectrum of hypatulin $\mathrm{A}(\mathbf{1})$ in $\mathrm{CD}_{3} \mathrm{OD}(125 \mathrm{MHz})$.

Figure S3. $\quad{ }^{13} \mathrm{C}$ NMR spectrum of hypatulin $\mathrm{A}(\mathbf{1})$ in $\mathrm{CD}_{3} \mathrm{OH}(125 \mathrm{MHz})$.

Figure S4. $\quad{ }^{1} \mathrm{H}-{ }^{1} \mathrm{H}$ COSY spectrum of hypatulin $\mathrm{A}(\mathbf{1})$ in $\mathrm{CD}_{3} \mathrm{OD}(500 \mathrm{MHz})$.

Figure S5. HSQC spectrum of hypatulin $\mathrm{A}(\mathbf{1})$ in $\mathrm{CD}_{3} \mathrm{OD}(500 \mathrm{MHz})$.

Figure S6. $\mathrm{HMBC}$ spectrum of hypatulin $\mathrm{A}(\mathbf{1})$ in $\mathrm{CD}_{3} \mathrm{OD}(500 \mathrm{MHz})$.

Figure S7. NOESY spectrum of hypatulin A (1) in $\mathrm{CD}_{3} \mathrm{OD}(500 \mathrm{MHz})$.

Figure S8. $\quad{ }^{1} \mathrm{H}$ NMR spectrum of hypatulin $\mathrm{B}(2)$ in $\mathrm{CD}_{3} \mathrm{OD}(500 \mathrm{MHz})$.

Figure S9. $\quad{ }^{13} \mathrm{C}$ NMR spectrum of hypatulin $\mathrm{B}(2)$ in $\mathrm{CD}_{3} \mathrm{OD}(125 \mathrm{MHz})$.

Figure S10. ${ }^{13} \mathrm{C}$ NMR spectrum of hypatulin $\mathrm{B}(2)$ in $\mathrm{CD}_{3} \mathrm{OH}(125 \mathrm{MHz})$.

Figure S11. $\quad{ }^{1} \mathrm{H}-{ }^{1} \mathrm{H}$ COSY spectrum of hypatulin B (2) in $\mathrm{CD}_{3} \mathrm{OD}(500 \mathrm{MHz})$.

Figure S12. HSQC spectrum of hypatulin $\mathrm{B}(2)$ in $\mathrm{CD}_{3} \mathrm{OD}(500 \mathrm{MHz})$.

Figure S13. HMBC spectrum of hypatulin $\mathrm{B}(2)$ in $\mathrm{CD}_{3} \mathrm{OD}(500 \mathrm{MHz})$.

Figure S14. NOESY spectrum of hypatulin $\mathrm{B}(2)$ in $\mathrm{CD}_{3} \mathrm{OD}(500 \mathrm{MHz})$.

Figure S15. $\quad{ }^{1} \mathrm{H}$ NMR spectrum of hypatulin B (1c) derived from hypatulin A (1) in $\mathrm{CD}_{3} \mathrm{OD}(500 \mathrm{MHz})$.

Table S1. $\quad{ }^{13} \mathrm{C}$ NMR data in $\mathrm{CD}_{3} \mathrm{OH}$ and $\mathrm{CD}_{3} \mathrm{OD}$ and deuterium-induced isotope shift for Hypatulins A (1) and B (2). 


\section{Experimental Section}

1. General Experimental Procedures. Optical rotations and IR spectra were recorded on a JASCO P-2200 digital polarimeter and a JASCO FT/IR-6200 spectrophotometer, respectively. UV spectra were recorded using a Hitachi UV-3900H spectrophotometer. CD spectrum was recorded on a JASCO J-1500 spectrophotometer. NMR spectra were measured by a Bruker AVANCE-500 instrument using tetramethylsilane as an internal standard. HRESIMS spectra were recorded on a Waters LCT PREMIER 2695.

2. Plant Material. Hypericum patulum was cultivated at Mori Botanical Garden in Tokushima, Japan, and collected in July 2013. The voucher specimen (HYP201307) was deposited at the herbarium of Graduate School Pharmaceutical Sciences, Tokushima University.

3. Extraction and Isolation. The dried leaves of Hypericum patulum $(1.48 \mathrm{~kg})$ was extracted with $\mathrm{MeOH}$ to afford the extract (629 g), which was partitioned with $n$-hexane and water. The $n$-hexane soluble fraction was subjected to silica gel column chromatography ( $n$-hexane/EtOAc, 9:1 to 8:2) to afford five fractions (frs. 1-5). Fr. 4 was loaded on a silica gel column ( $n$-hexane/ $\mathrm{CHCl}_{3}, 1: 1$ to $0: 1$ ) to give six fractions (frs. 4.1-6). Fr.4.5 was separated by an ODS column $\left(\mathrm{MeOH} / \mathrm{H}_{2} \mathrm{O}, 8: 2\right.$ to $\left.10: 0\right)$ and a silica gel column $\left(\mathrm{CHCl}_{3}\right)$ chromatographies, and purified using ODS HPLC (YMC-Triart C18, YMC CO., LTD, 10 × $250 \mathrm{~mm}, \mathrm{MeOH} / \mathrm{H}_{2} \mathrm{O}$, 9:1) to give hypatulins $\mathrm{A}(\mathbf{1}, 36.9 \mathrm{mg})$ and $\mathrm{B}(\mathbf{2}, 3.6 \mathrm{mg})$.

3.1. Hypatulin A (1): colorless amorphous solid; $[\alpha]^{25}+40.4(c 0.046, \mathrm{MeOH})$; IR $(\mathrm{KBr}) v_{\max }$ $3455,1786,1738$, and $1672 \mathrm{~cm}^{-1}$; UV (MeOH) $\lambda_{\max } 245$ ( $\varepsilon$ 9200) nm; ECD (MeOH) $\Delta \varepsilon(\mathrm{nm})-$ 1.1 (334), -1.0 (281), and +5.1 (243); ${ }^{1} \mathrm{H}$ and ${ }^{13} \mathrm{C}$ NMR (Table 1); HRESIMS: $m / z$ 511.2807 $[\mathrm{M}+\mathrm{Na}]^{+}$(calcd for $\mathrm{C}_{32} \mathrm{H}_{40} \mathrm{O}_{4} \mathrm{Na}, 511.2824$ ).

3.2. Hypatulin B (2): colorless amorphous solid; $[\alpha]^{25}{ }_{\mathrm{D}}+27.0(c 0.17, \mathrm{MeOH})$; $\mathrm{IR}(\mathrm{KBr}) v_{\max }$ 3507, 1738, and $1672 \mathrm{~cm}^{-1}$; UV (MeOH) $\lambda_{\max } 243$ ( $\varepsilon$ 10500); ${ }^{1} \mathrm{H}$ and ${ }^{13} \mathrm{C}$ NMR (Table 1); HRESIMS: $m / z 543.3064[\mathrm{M}+\mathrm{Na}]^{+}$(calcd for $\mathrm{C}_{33} \mathrm{H}_{44} \mathrm{O}_{5} \mathrm{Na}, 543.3086$ ).

4. Calculations. Conformational searches and DFT calculations were carried out on Spartan 
$14 \operatorname{program}^{\mathrm{S} 1}$ and Gaussian 09 program, ${ }^{\mathrm{S} 2}$ respectively. Possible enantiomers 1a $(1 S, 3 R, 4 R, 6 R, 7 R, 8 S)$ and $\mathbf{1 b}(1 R, 3 S, 4 S, 6 S, 7 S, 8 R)$ were submitted to conformational searches at the Molecular Mechanics (MMFF94s). The initial stable conformers for 1a and 1b with Boltzmann distributions over 1\% (13 and 12 conformers, respectively) were further optimized by DFT calculations at the $\mathrm{B} 3 \mathrm{LYP} / 6-31 \mathrm{G}(\mathrm{d})$ level in the presence of $\mathrm{MeOH}$ with a polarizable continuum model (PCM). The stable conformers for 1a and 1b with Boltzmann distributions over 1\% (12 and 11 conformers, respectively) were subjected to TDDFT calculations at the B3LYP/6-31G(d) level in the presence of $\mathrm{MeOH}$ with a PCM. The resultant rotatory strengths of the lowest 30 excited states for each conformer were converted into Gaussian-type curves with half-bands $(0.3 \mathrm{eV})$ using SpecDis v1.61 ${ }^{\mathrm{S} 3}$ The calculated CD spectra for $1 \mathbf{a}$ and $\mathbf{1 b}$ were composed after correction based on the Boltzmann distribution of the stable conformers and blue-shifted by $15 \mathrm{~nm}$, respectively.

\section{Chemical conversion of hypatulin A (1) into hypatulin B (1c).}

A mixture of hypatulin A (1, $5 \mathrm{mg})$ and 4-dimethylaminopyridine $(12 \mathrm{mg})$ in $\mathrm{MeOH}(5 \mathrm{~mL})$ was stirred at $\mathrm{rt}$ for $12 \mathrm{~h}$. The reaction mixture was diluted by water $(30 \mathrm{~mL})$ and extracted with EtOAc $(20 \mathrm{~mL} \times 3)$. The EtOAc layer was washed with $1 \mathrm{M} \mathrm{HCl}$ and brine, and concentrated to give a residue, which was purified by ODS HPLC (YMC-Triart C18, 10x250 mm, MeOH: $\mathrm{H}_{2} \mathrm{O}$, 9:1) to give hypatulin B (1c, $2.3 \mathrm{mg})$.

5.1. Hypatulin B (1c) derived from hypatulin A (1): colorless amorphous solid; $[\alpha]_{D}^{25}+25.4(c$ $0.11, \mathrm{MeOH}$ ); ${ }^{1} \mathrm{H}$ NMR spectrum of 1c (Fig. S15) was identical to that of natural hypatulin B (2) (Fig. S8).

\section{Antimicrovial Assay.}

Test microorganisms. Seven clinical isolates of methicillin-resistant Staphylococcus aureus (MRSA) strains, three clinical isolates of methicillin-sensitive $S$. aureus (MSSA) strains, S. aureus 209P and Smith, one Bacillus subtilis strain, and four Escherichia coli strains were used in this study. All the strains of microorganisms used in this work were kindly supplied by Dr. C. Sano, the School of Medicine, Shimane University (Shimane, Japan).

Susceptibility testing. The MICs were determined by broth microdilution method in 96-well microtiter plates with cation-supplemented Mueller-Hinton broth (CAMHB; 
$\mathrm{Ca}^{2+}, 25 \mu \mathrm{g} / \mathrm{mL} ; \mathrm{Mg}^{2+}, 12.5 \mu \mathrm{g} / \mathrm{mL}$; Becton Dickinson, Sparks, MD) according to the current guidelines of the Clinical and Laboratory Standards Institute (CLSI). All the strains of microorganisms were inoculated at a final bacterial density of about $5 \times 10^{5}$ $\mathrm{CFU} / \mathrm{ml}$. Then, S. aureus strains were incubated at $35^{\circ} \mathrm{C}$ for $20 \mathrm{~h}$, and B. subtilis and $E$. coli strains were incubated at $37^{\circ} \mathrm{C}$ for $20 \mathrm{~h}$ before the MICs were determined.

\section{References.}

(S1) Spartan 14, Wavefunction Inc., Irvine, CA.

(S2) Gaussian 09, Revision C.01, Frisch, M. J.; Trucks, G. W.; Schlegel, H. B.; Scuseria, G. E.; Robb, M. A.; Cheeseman, J. R.; Scalmani, G.; Barone, V. Mennucci, B.; Petersson, G. A.; Nakatsuji, H.; Caricato, M.; Li, X.; Hratchian, H. P.; Izmaylov, A. F.; Bloino, J.; Zheng, G.; Sonnenberg, J. L.; Hada, M.; Ehara, M.; Toyota, K.; Fukuda, R.; Hasegawa, J.; Ishida, M.; Nakajima, T.; Honda, Y.; Kitao, O.; Nakai, H.; Vreven, T.; Montgomery, Jr., J. A.; Peralta, J. E.; Ogliaro, F.; Bearpark, M.; Heyd, J. J.; Brothers, E.; Kudin, K. N.; Staroverov, V. N.; Keith, T.; Kobayashi, R.; Normand, J.; Raghavachari, K.; Rendell, A.; Burant, J. C.; Iyengar, S. S.; Tomasi, J.; Cossi, M.; Rega, N.; Millam, J. M.; Klene, M.; Knox, J. E.; Cross, J. B.; Bakken, V.; Adamo, C.; Jaramillo, J.; Gomperts, R.; Stratmann, R. E.; Yazyev, O.; Austin, A. J.; Cammi, R.; Pomelli, C.; Ochterski, J. W.; Martin, R. L.; Morokuma, K.; Zakrzewski, V. G.; Voth, G. A.; Salvador, P.; Dannenberg, J. J.; Dapprich, S.; Daniels, A. D.; Farkas, O.; Foresman, J. B.; Ortiz, J. V.; Cioslowski, J.; and Fox, D. J. Gaussian, Inc., Wallingford, CT, 2010.

(S3) Bruhn, T.; Schaumlöffel, A.; Hemberger, Y.; Bringmann, G. SpecDis, Version 1.61, University of Wuerzburg, Germany, 2013. 
Figure S1. $\quad{ }^{1} \mathrm{H}$ NMR spectrum of hypatulin $\mathrm{A}(\mathbf{1})$ in $\mathrm{CD}_{3} \mathrm{OD}(500 \mathrm{MHz})$.



Figure S2. $\quad{ }^{13} \mathrm{C}$ NMR spectrum of hypatulin $\mathrm{A}(\mathbf{1})$ in $\mathrm{CD}_{3} \mathrm{OD}(125 \mathrm{MHz})$.

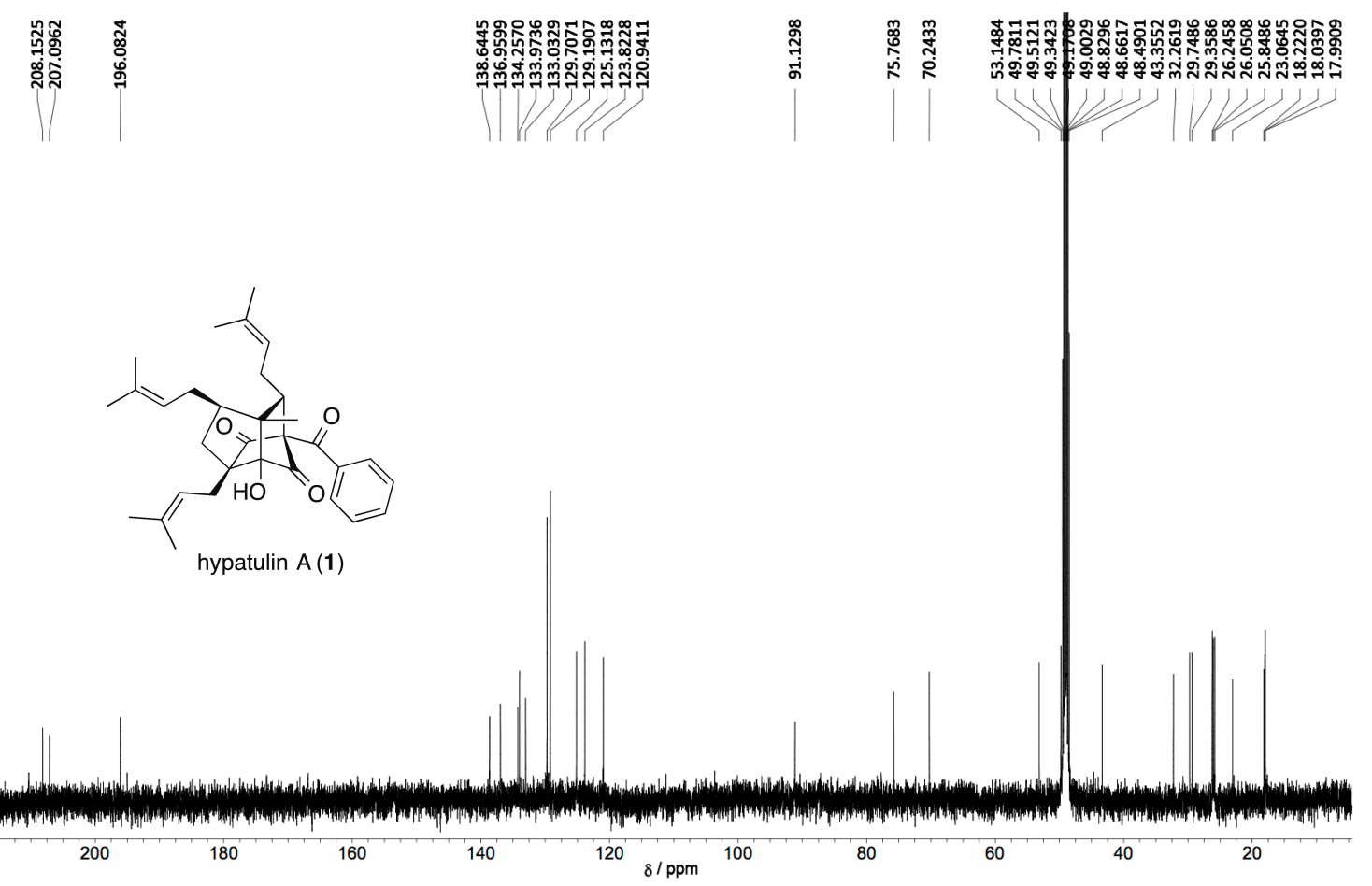


Figure S3. $\quad{ }^{13} \mathrm{C}$ NMR spectrum of hypatulin $\mathrm{A}(\mathbf{1})$ in $\mathrm{CD}_{3} \mathrm{OH}(125 \mathrm{MHz})$.

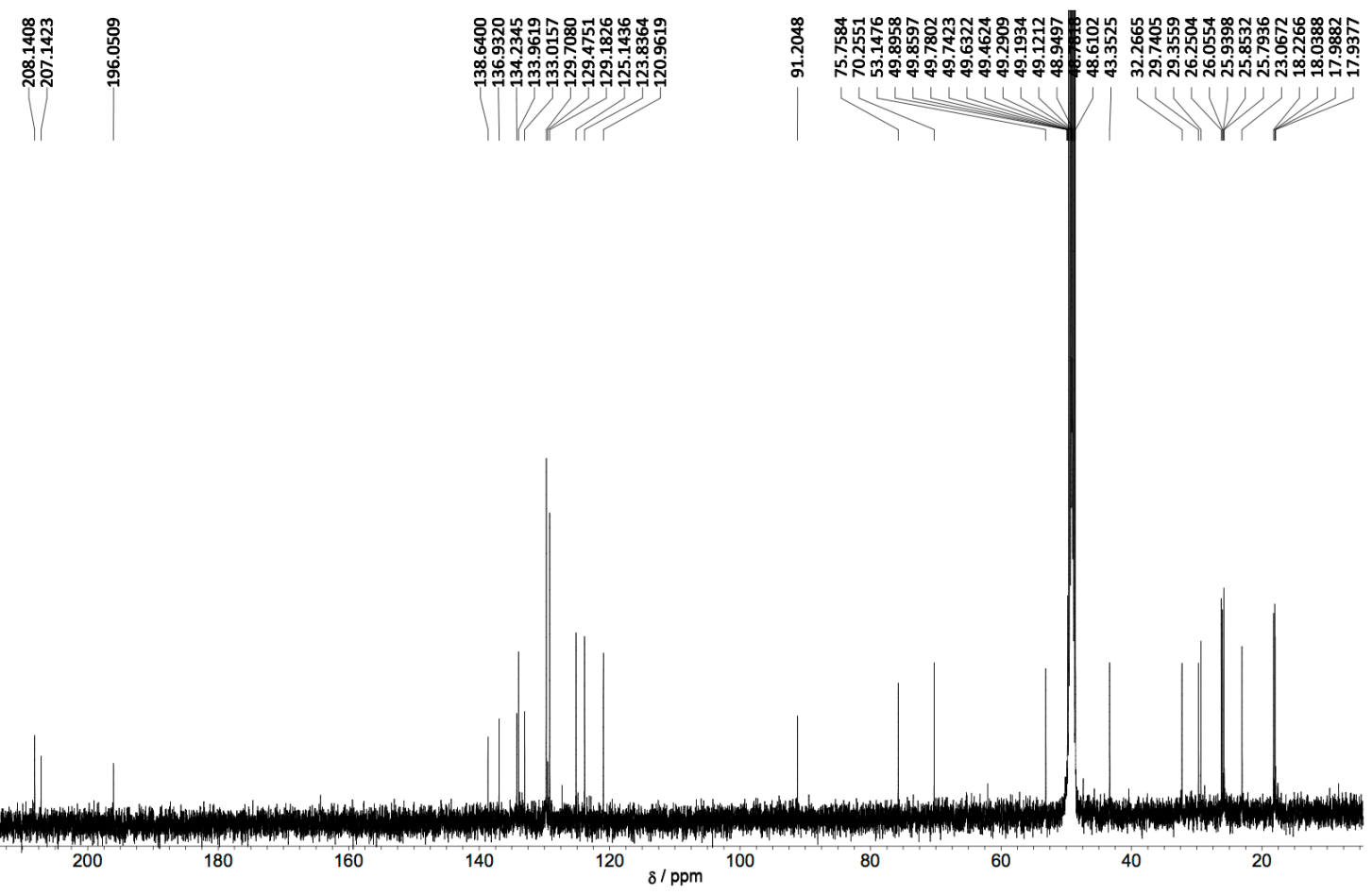

Figure S4. $\quad{ }^{1} \mathrm{H}-{ }^{1} \mathrm{H}$ COSY spectrum of hypatulin $\mathrm{A}(\mathbf{1})$ in $\mathrm{CD}_{3} \mathrm{OD}(500 \mathrm{MHz})$.

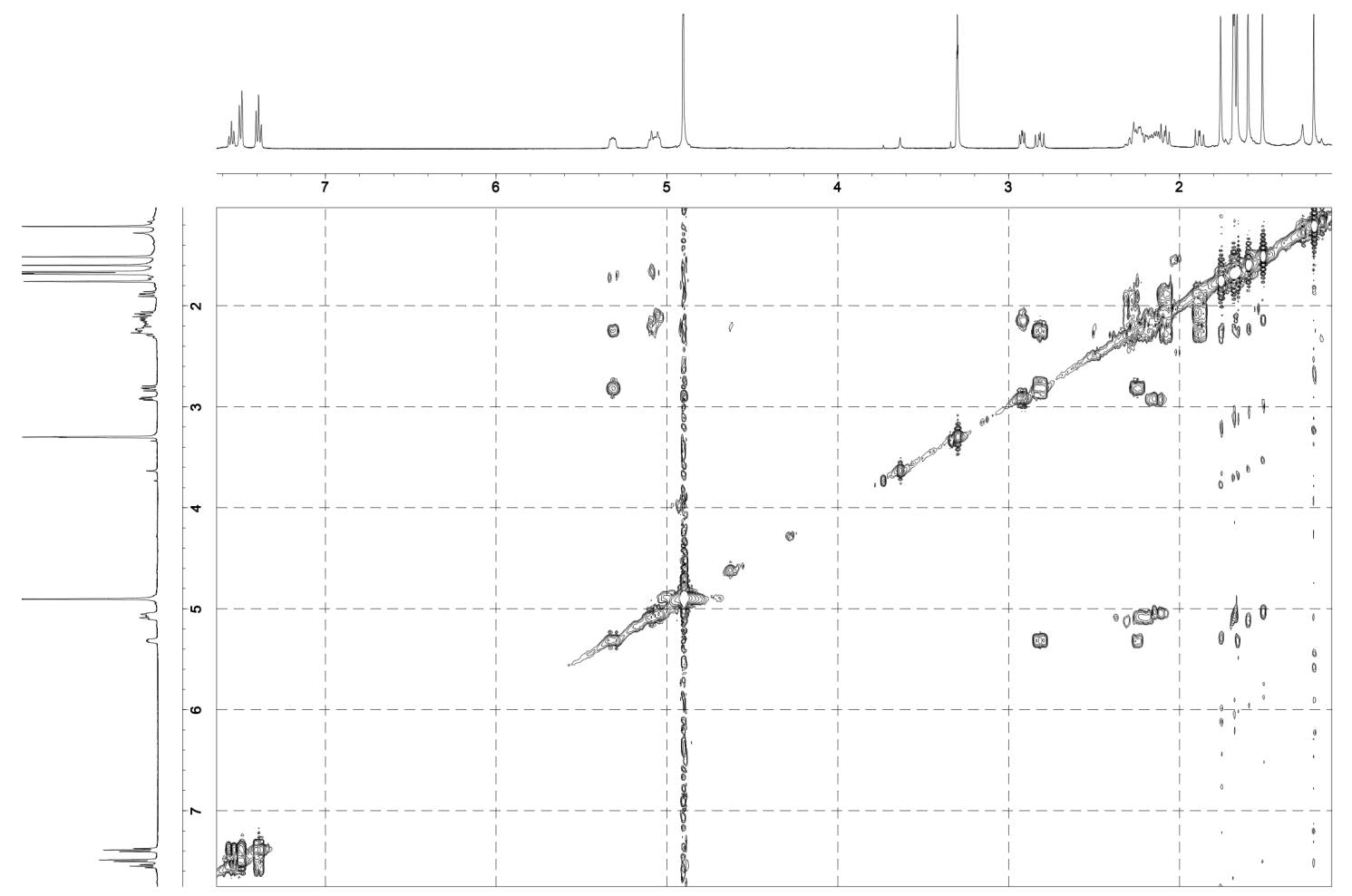


Figure S5. HSQC spectrum of hypatulin $\mathrm{A}(\mathbf{1})$ in $\mathrm{CD}_{3} \mathrm{OD}(500 \mathrm{MHz})$.

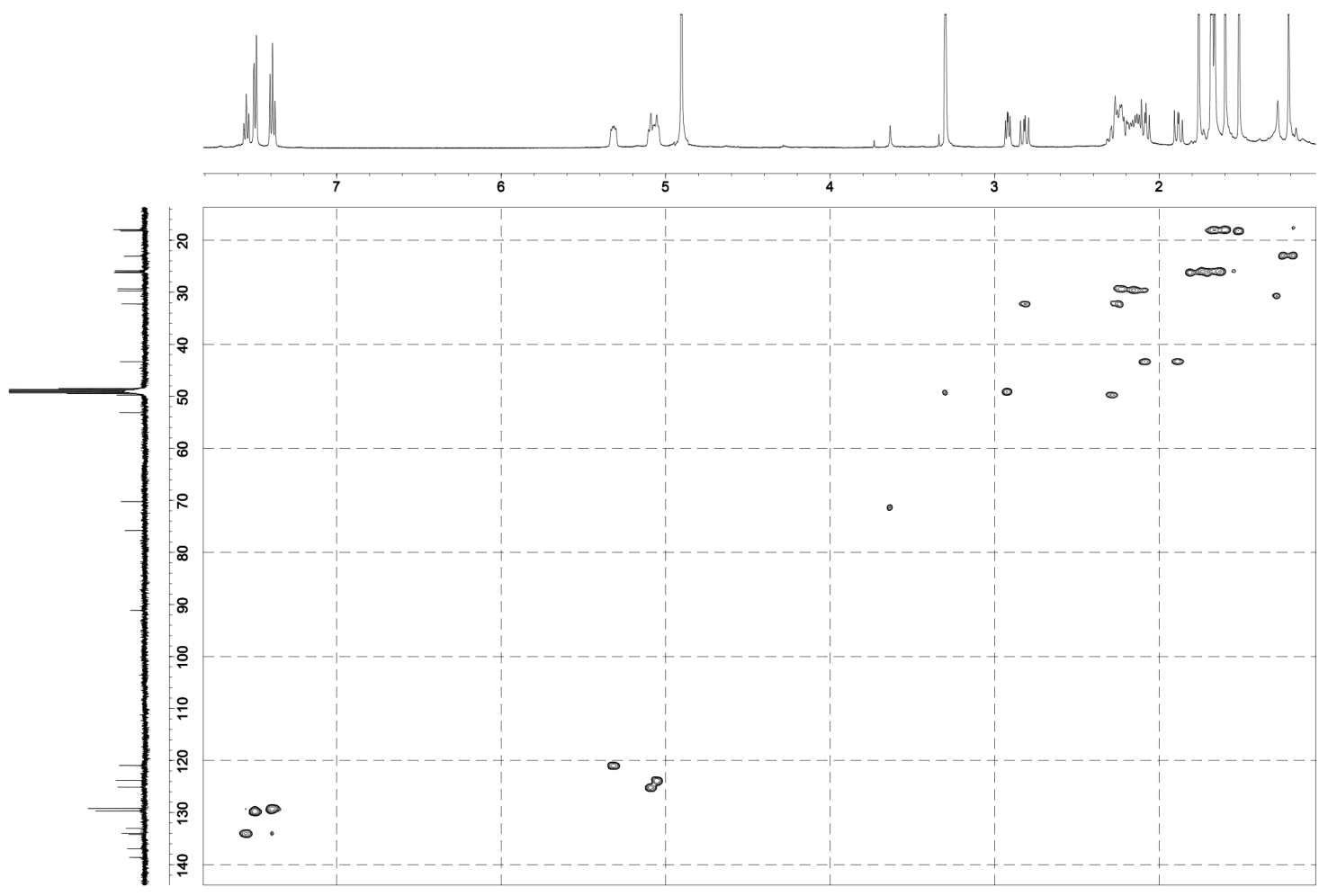

Figure S6. HMBC spectrum of hypatulin $\mathrm{A}(\mathbf{1})$ in $\mathrm{CD}_{3} \mathrm{OD}(500 \mathrm{MHz})$.

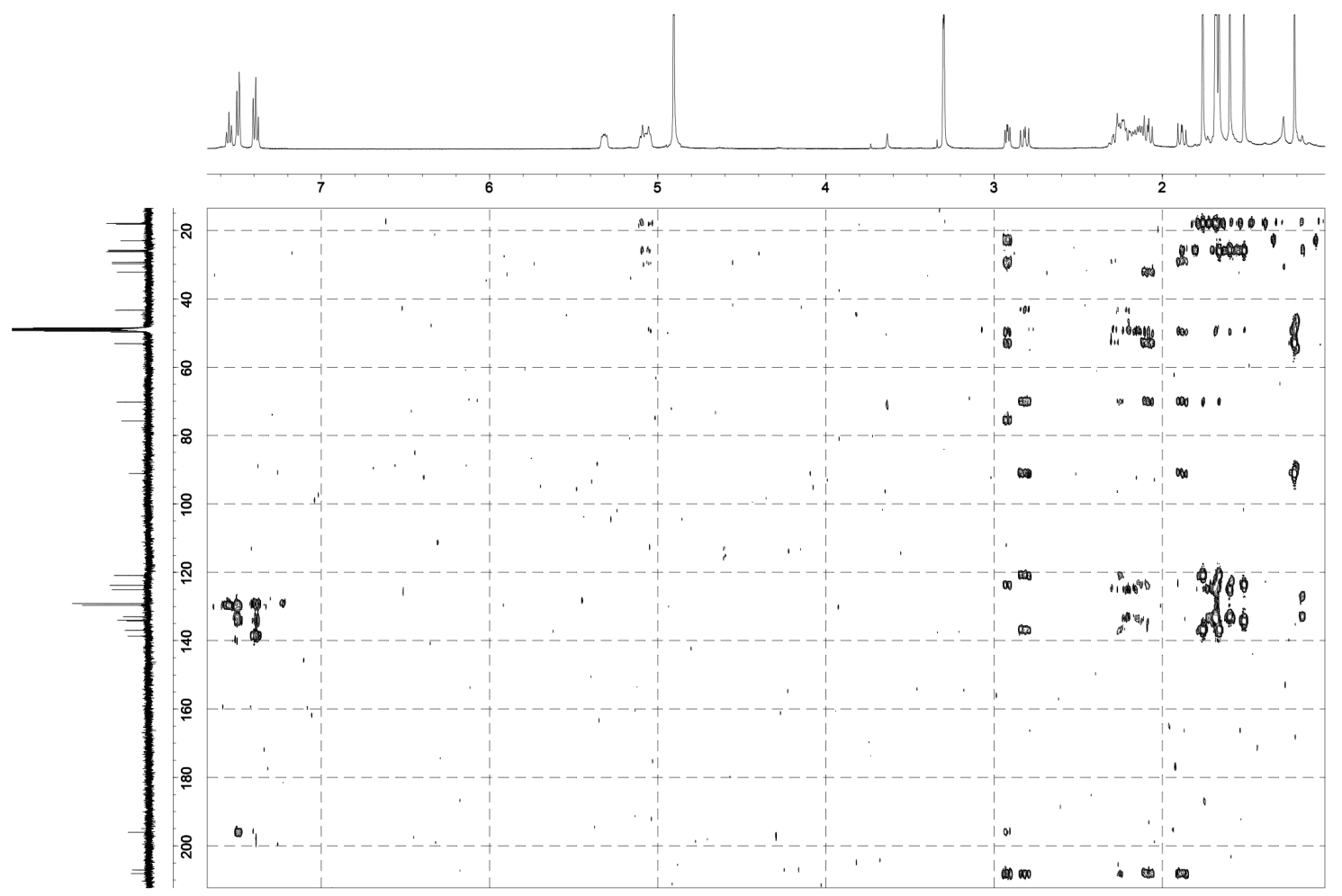


Figure S7. NOESY spectrum of hypatulin $\mathrm{A}(\mathbf{1})$ in $\mathrm{CD}_{3} \mathrm{OD}(500 \mathrm{MHz})$.

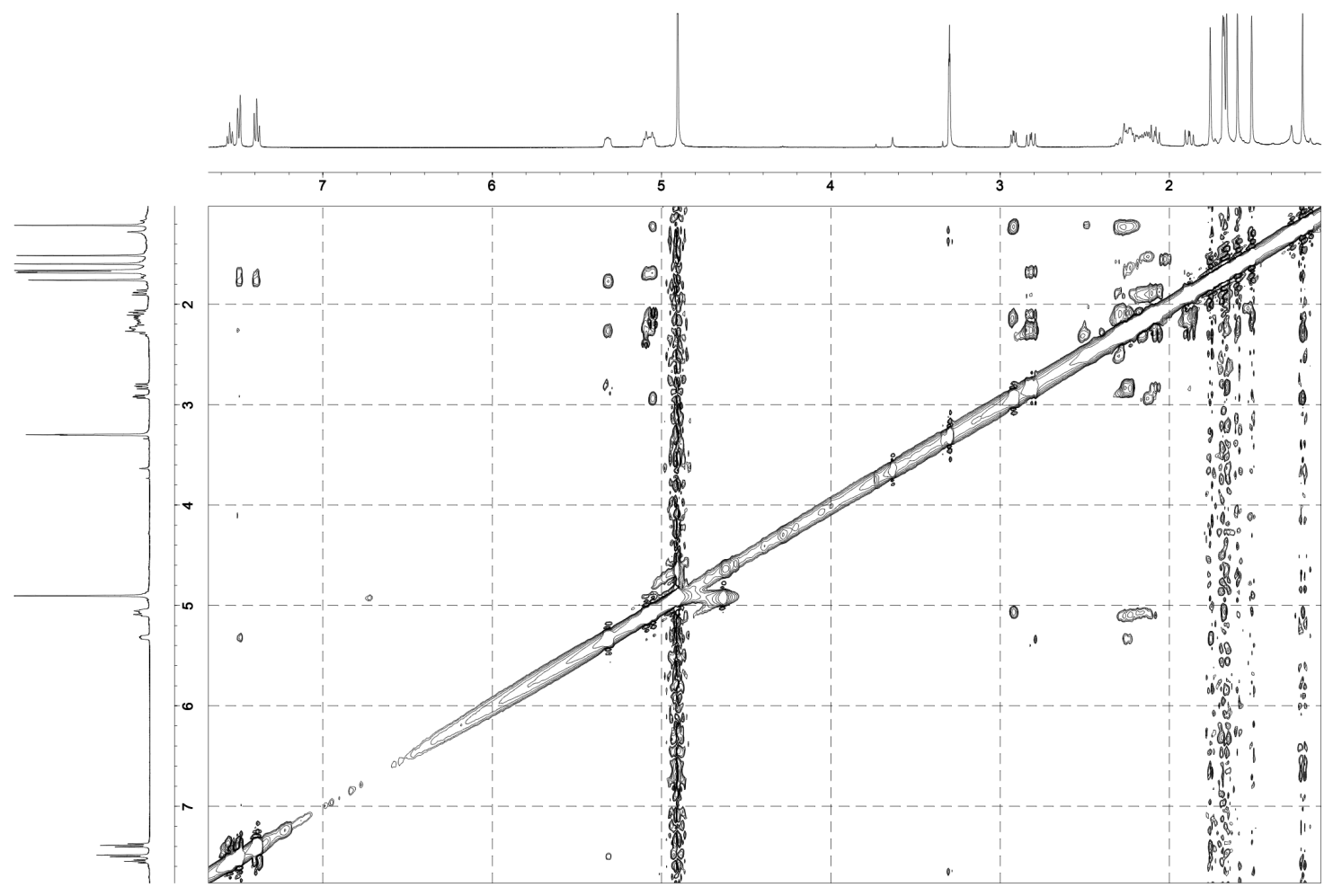

Figure S8. $\quad{ }^{1} \mathrm{H}$ NMR spectrum of hypatulin B (2) in $\mathrm{CD}_{3} \mathrm{OD}(500 \mathrm{MHz})$.

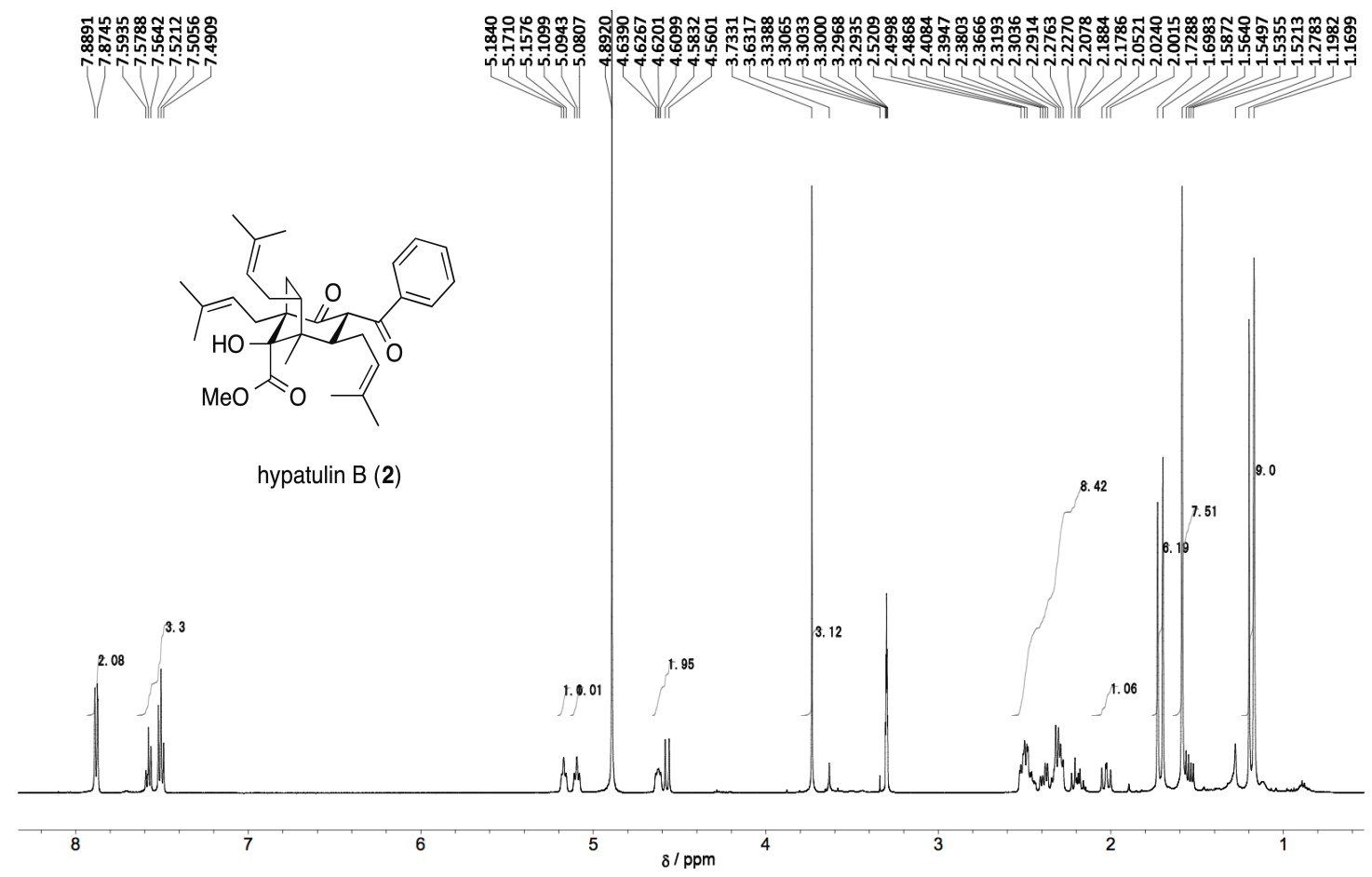


Figure S9. $\quad{ }^{13} \mathrm{C}$ NMR spectrum of hypatulin $\mathrm{B}(2)$ in $\mathrm{CD}_{3} \mathrm{OD}(125 \mathrm{MHz})$.

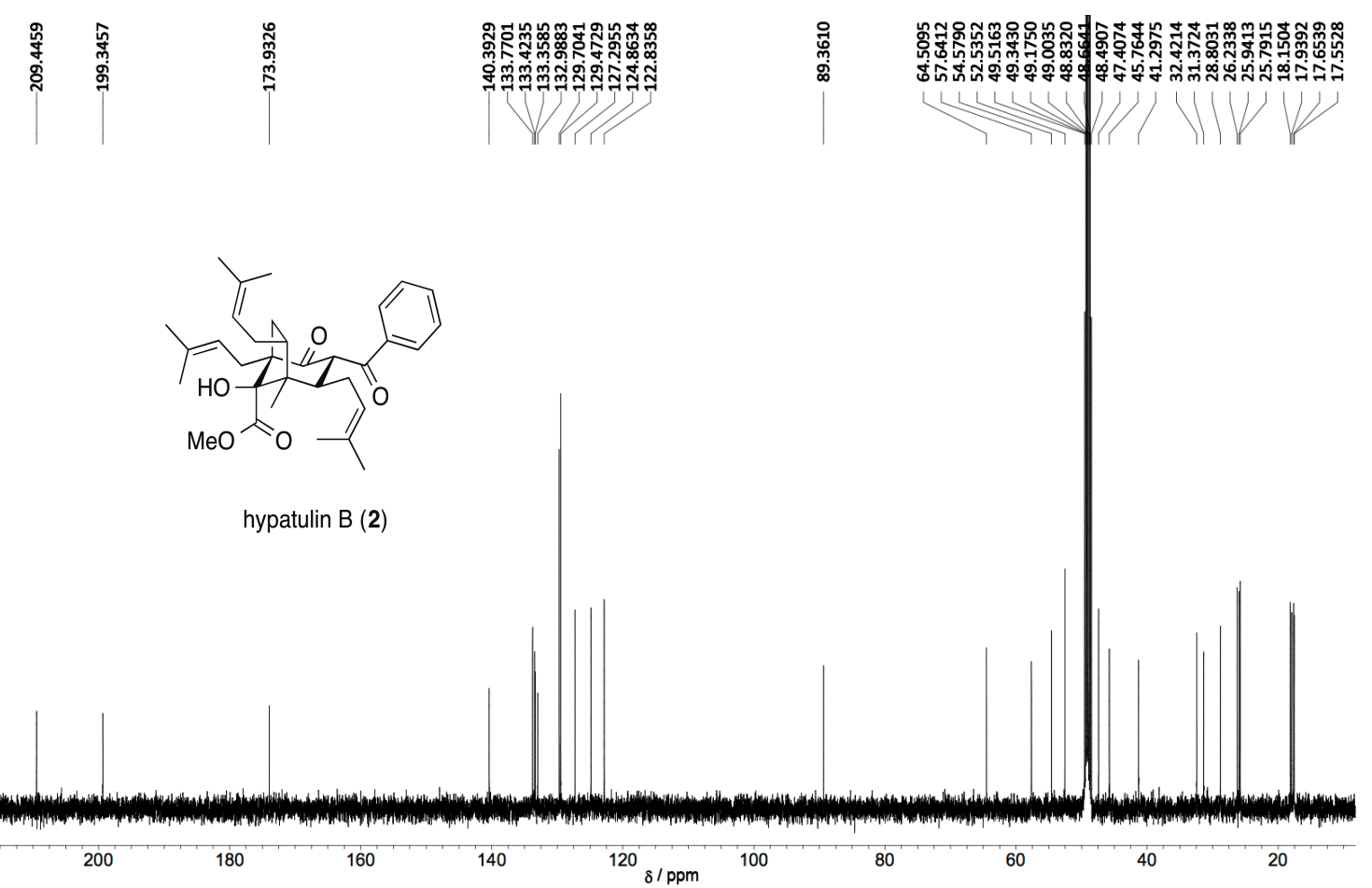

Figure S10. ${ }^{13} \mathrm{C}$ NMR spectrum of hypatulin $\mathrm{B}(2)$ in $\mathrm{CD}_{3} \mathrm{OH}(125 \mathrm{MHz})$.
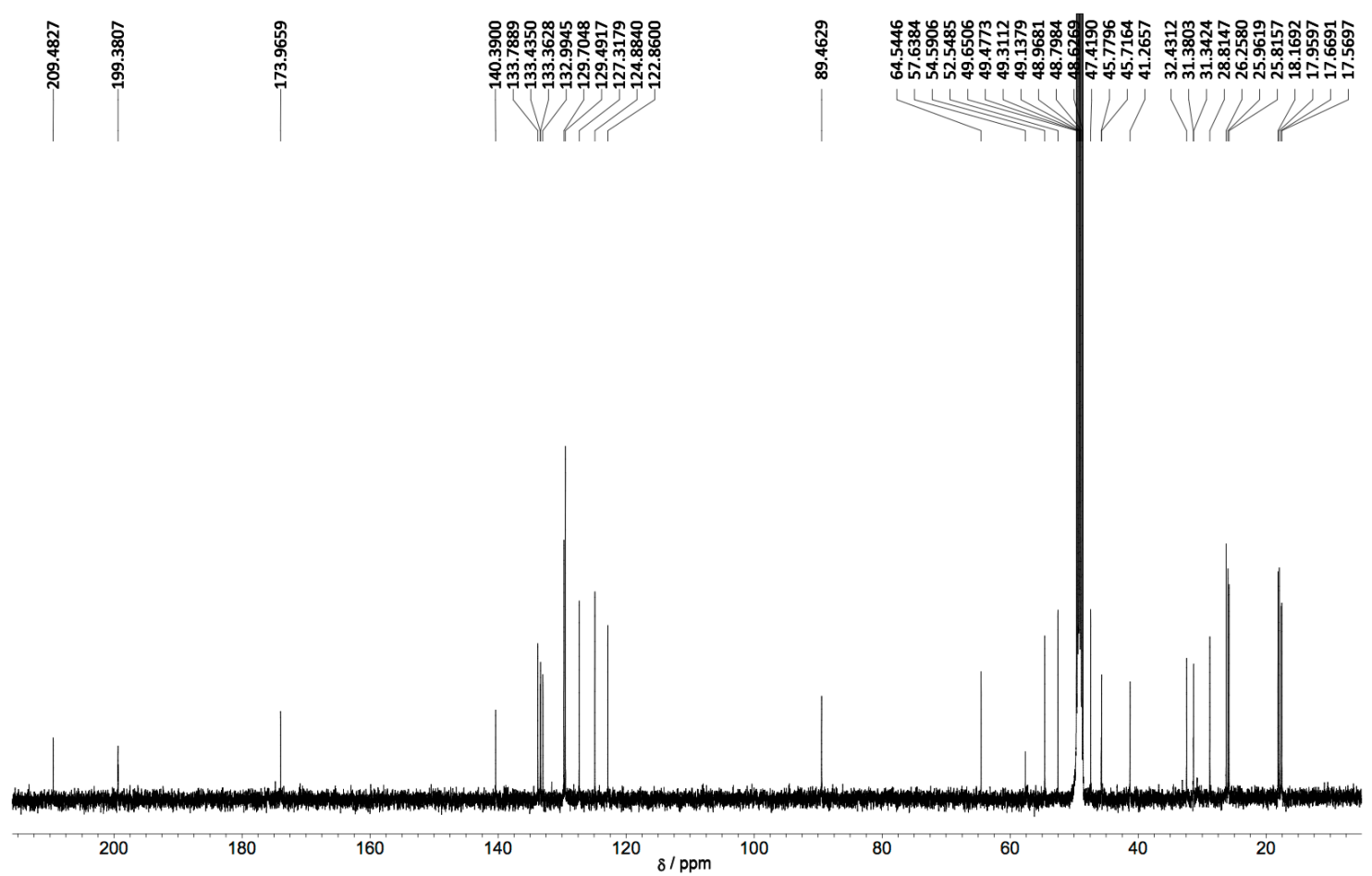
Figure S11. $\quad{ }^{1} \mathrm{H}-{ }^{1} \mathrm{H}$ COSY spectrum of hypatulin B (2) in $\mathrm{CD}_{3} \mathrm{OD}(500 \mathrm{MHz})$.

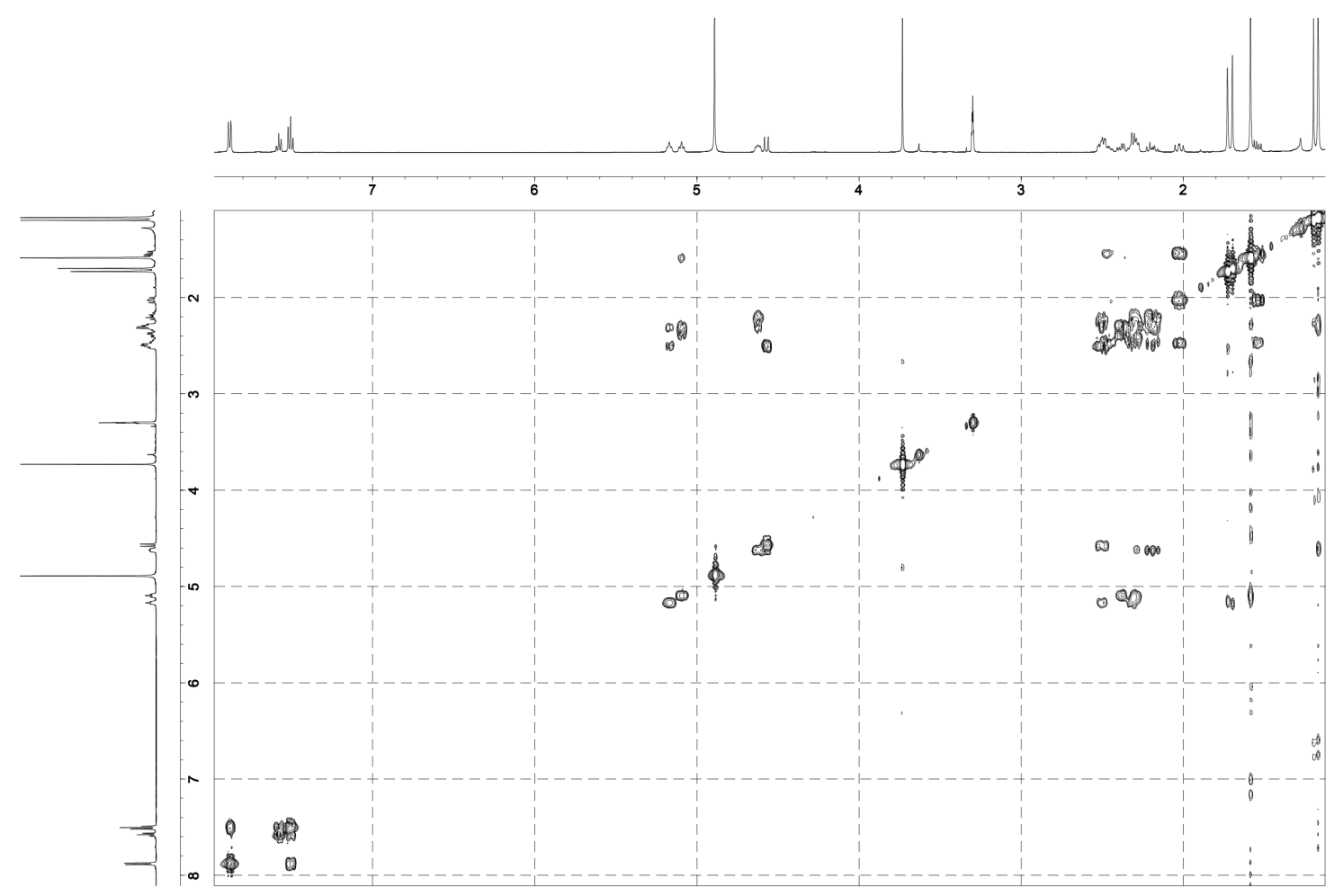

Figure S12. HSQC spectrum of hypatulin $\mathrm{B}(2)$ in $\mathrm{CD}_{3} \mathrm{OD}(500 \mathrm{MHz})$.

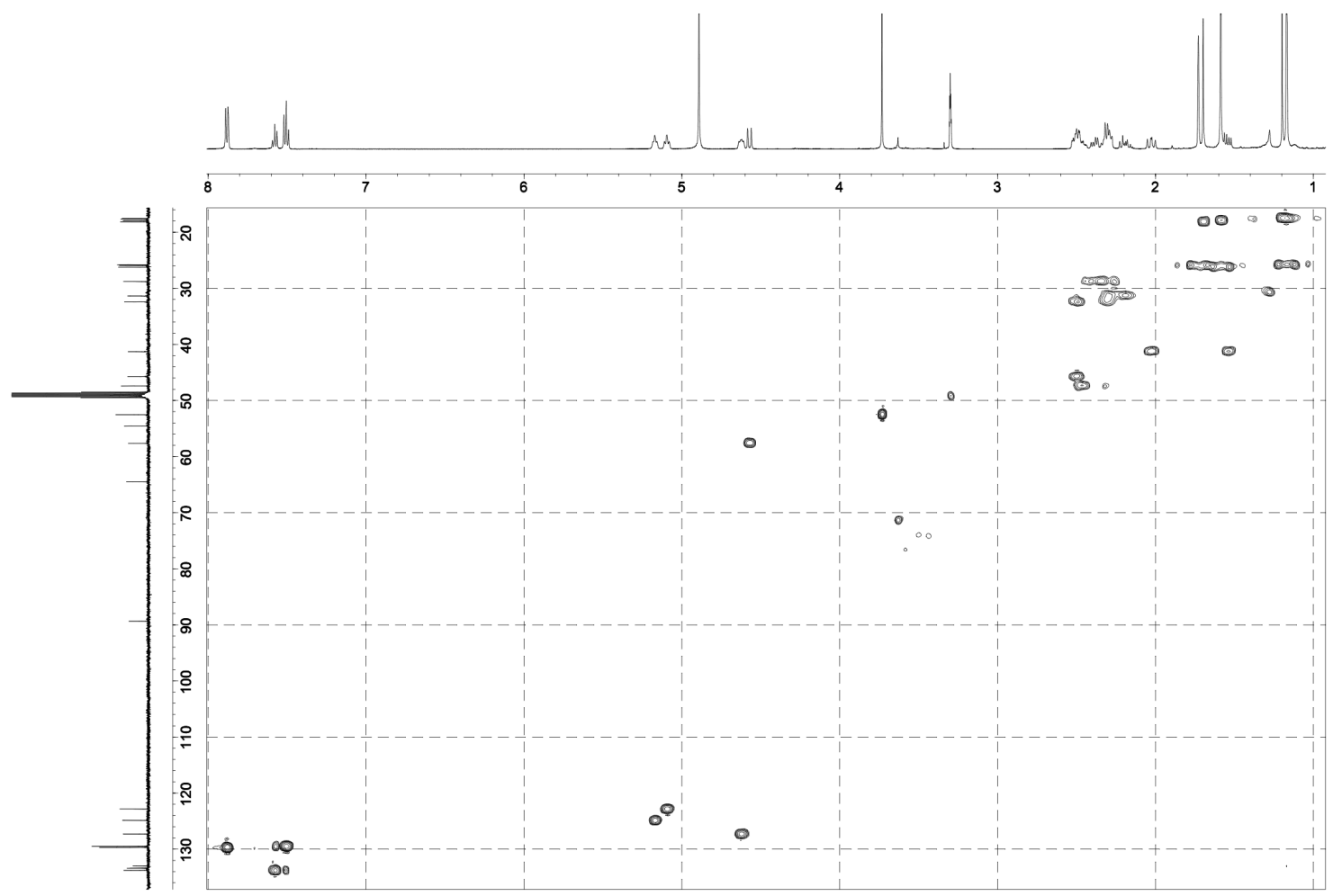


Figure $\mathrm{S} 13$. $\mathrm{HMBC}$ spectrum of hypatulin $\mathrm{B}(2)$ in $\mathrm{CD}_{3} \mathrm{OD}(500 \mathrm{MHz})$.

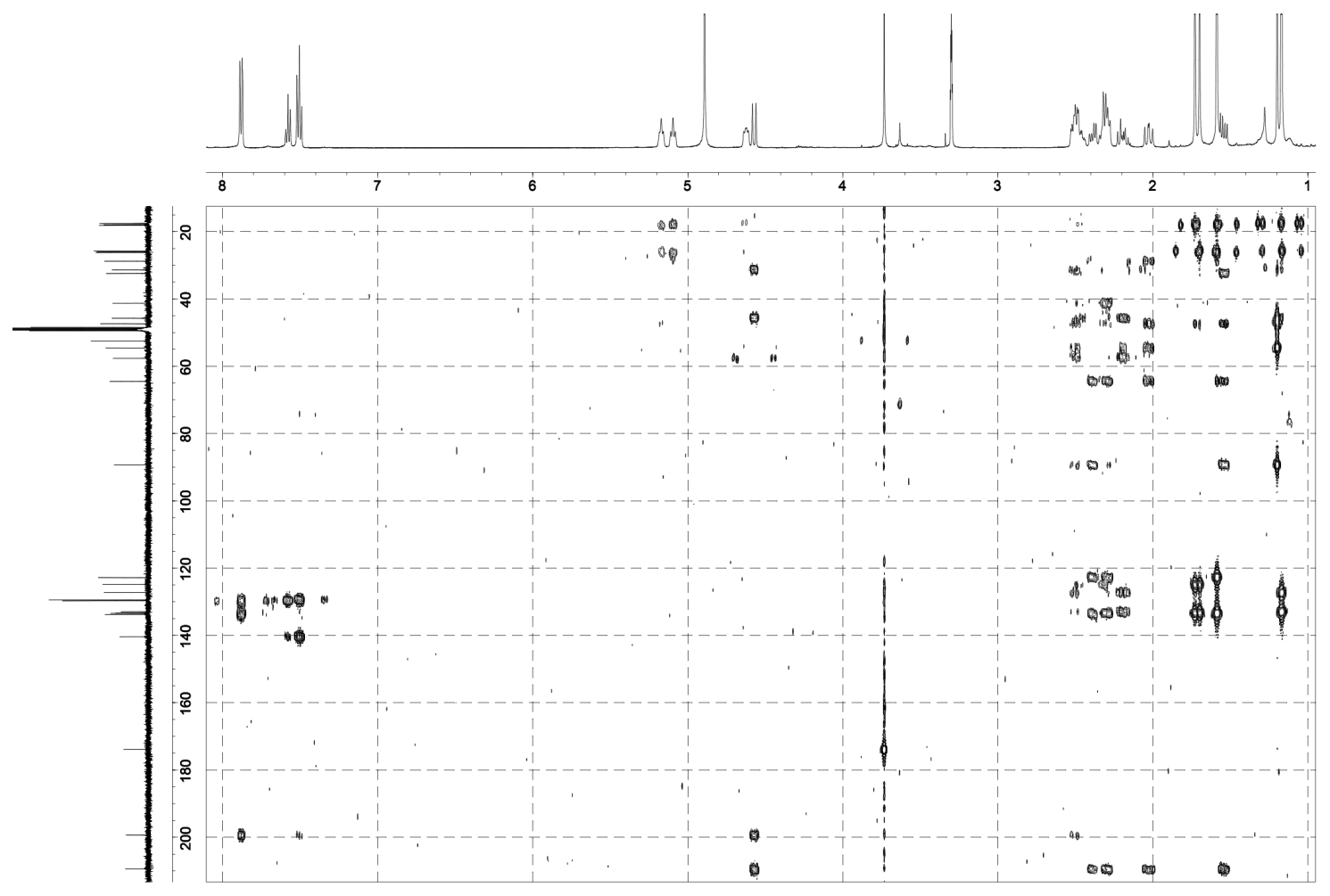

Figure S14. NOESY spectrum of hypatulin B (2) in $\mathrm{CD}_{3} \mathrm{OD}(500 \mathrm{MHz})$.

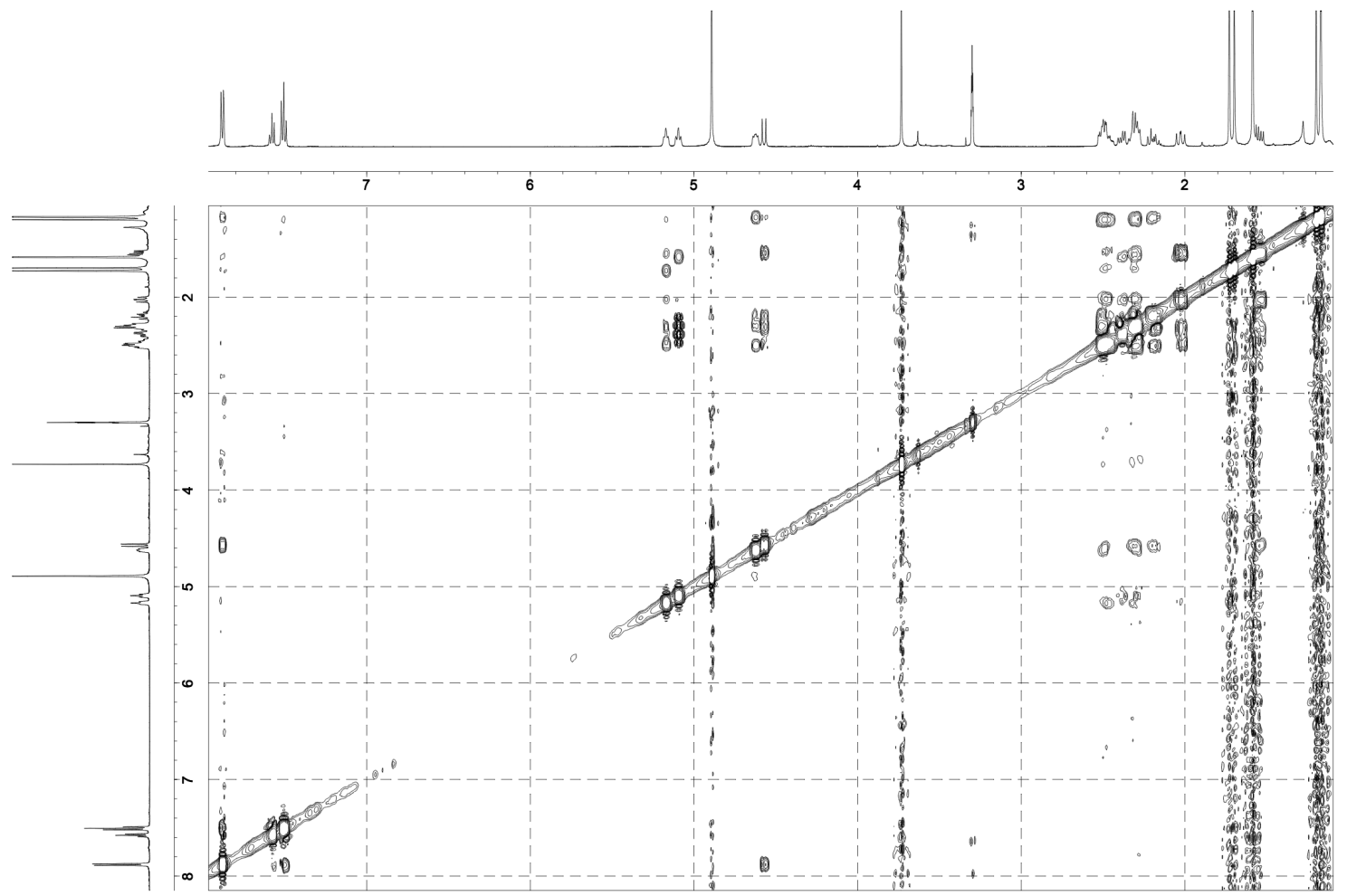


Figure S15. $\quad{ }^{1} \mathrm{H}$ NMR spectrum of hypatulin B (1c) derived from hypatulin A (1) in $\mathrm{CD}_{3} \mathrm{OD}(500 \mathrm{MHz})$.

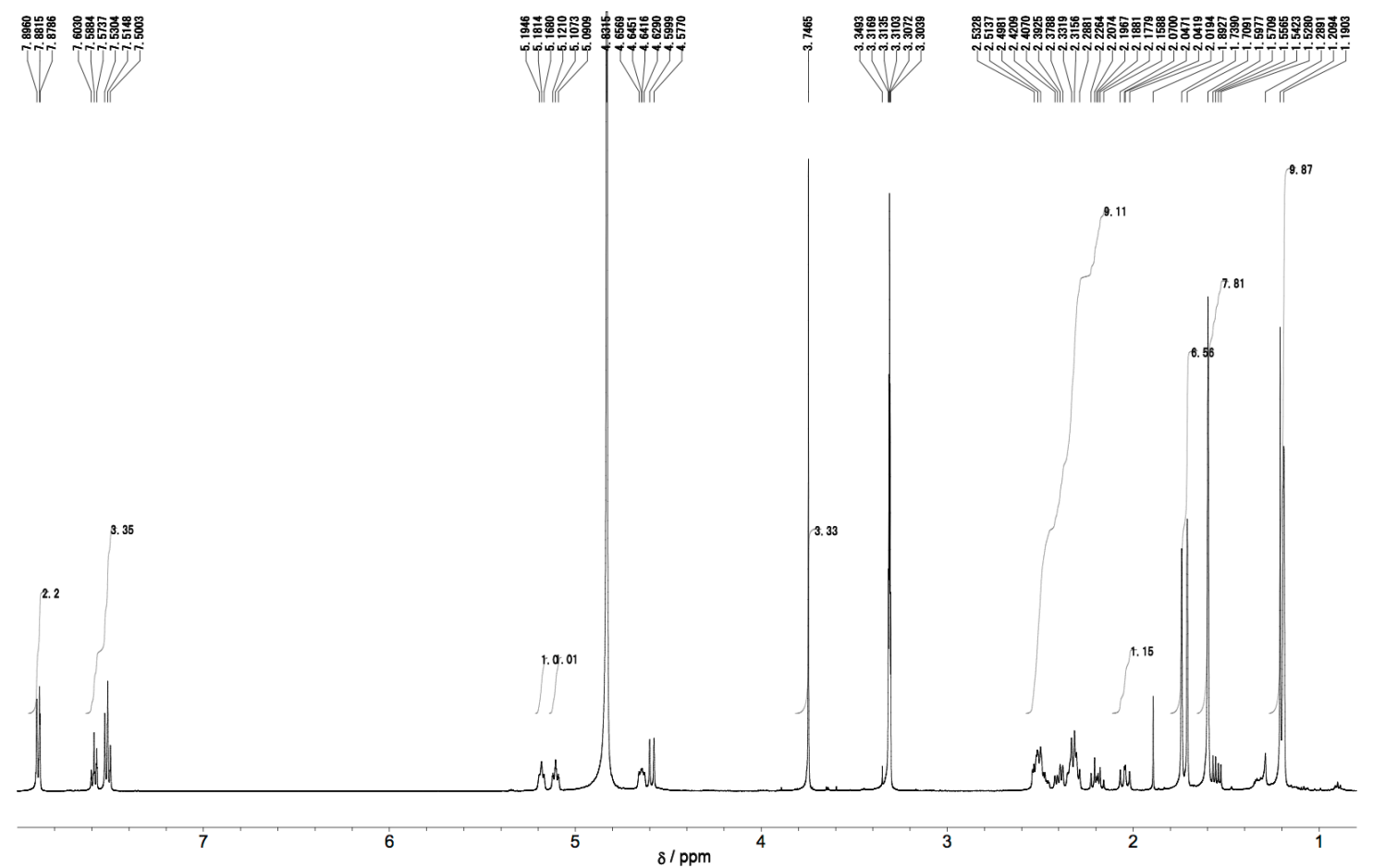


Table S1. ${ }^{13} \mathrm{C}$ NMR Data in $\mathrm{CD}_{3} \mathrm{OH}$ and $\mathrm{CD}_{3} \mathrm{OD}$ and Deuterium-induced Isotope Shifts for Hypatulins A (1) and B (2).

\begin{tabular}{|c|c|c|c|c|c|c|}
\hline \multirow[b]{2}{*}{ position } & \multicolumn{3}{|c|}{1} & \multicolumn{3}{|c|}{2} \\
\hline & $\begin{array}{r}{ }^{13} \mathrm{C} \\
\left(\mathrm{CD}_{3} \mathrm{OH}\right)\end{array}$ & $\begin{array}{c}{ }^{13} \mathrm{C} \\
\left(\mathrm{CD}_{3} \mathrm{OD}\right)\end{array}$ & $\begin{array}{c}\Delta \delta \\
\left(\mathrm{CD}_{3} \mathrm{OH}-\mathrm{CD}_{3} \mathrm{OD}\right)\end{array}$ & $\begin{array}{c}{ }^{13} \mathrm{C} \\
\left(\mathrm{CD}_{3} \mathrm{OH}\right)\end{array}$ & $\begin{array}{c}{ }^{13} \mathrm{C} \\
\left(\mathrm{CD}_{3} \mathrm{OD}\right)\end{array}$ & $\begin{array}{c}\Delta \delta \\
\left(\mathrm{CD}_{3} \mathrm{OH}-\mathrm{CD} \mathrm{OD}_{3} \mathrm{OD}\right)\end{array}$ \\
\hline 1 & 75.76 & 75.77 & -0.01 & 57.63 & 57.64 & -0.01 \\
\hline 2 & 207.14 & 207.10 & 0.04 & 173.96 & 173.93 & 0.03 \\
\hline 3 & 91.20 & 91.13 & 0.07 & 89.45 & 89.36 & 0.09 \\
\hline 4 & 70.26 & 70.24 & 0.02 & 64.53 & 64.51 & 0.02 \\
\hline 5 & 43.35 & 43.36 & -0.01 & 41.26 & 41.30 & -0.04 \\
\hline 6 & $49.9^{\mathrm{a}}$ & $49.8^{\mathrm{a}}$ & - & 47.41 & 47.41 & 0.00 \\
\hline 7 & 53.15 & 53.15 & 0.00 & 54.58 & 54.58 & 0.00 \\
\hline 8 & $49.2^{\mathrm{a}}$ & $49.2^{\mathrm{a}}$ & - & 45.77 & 45.76 & 0.01 \\
\hline 9 & 208.14 & 208.15 & -0.01 & 209.47 & 209.45 & 0.02 \\
\hline 10 & 23.07 & 23.06 & 0.01 & 17.56 & 17.55 & 0.01 \\
\hline 11 & 32.27 & 32.26 & 0.01 & 28.8 & 28.80 & 0.00 \\
\hline 12 & 120.96 & 120.94 & 0.02 & 122.85 & 122.84 & 0.01 \\
\hline 13 & 136.93 & 136.96 & -0.03 & $133.42^{\mathrm{b}}$ & $133.42^{\mathrm{c}}$ & 0.00 \\
\hline 14 & 17.99 & 17.99 & 0.00 & 17.95 & 17.94 & 0.01 \\
\hline 15 & 26.25 & 26.25 & 0.00 & 26.25 & 26.23 & 0.02 \\
\hline 16 & 29.36 & 29.36 & 0.00 & 32.42 & 32.42 & 0.00 \\
\hline 17 & 125.14 & 125.13 & 0.01 & 124.87 & 124.86 & 0.01 \\
\hline 18 & 133.02 & 133.03 & -0.01 & $133.35^{\mathrm{b}}$ & $133.36^{\mathrm{c}}$ & -0.01 \\
\hline 19 & 18.04 & 18.04 & 0.00 & 18.16 & 18.15 & 0.01 \\
\hline 20 & 25.85 & 25.85 & 0.00 & 25.95 & 25.94 & 0.01 \\
\hline 21 & 29.74 & 29.75 & -0.01 & 31.37 & 31.37 & 0.00 \\
\hline 22 & 123.84 & 123.82 & 0.02 & 127.31 & 127.30 & 0.01 \\
\hline 23 & 134.23 & 134.26 & -0.03 & 132.98 & 132.99 & -0.01 \\
\hline 24 & 18.23 & 18.22 & 0.01 & 17.66 & 17.65 & 0.01 \\
\hline 25 & 26.06 & 26.05 & 0.01 & 25.80 & 25.79 & 0.01 \\
\hline 26 & 196.05 & 196.08 & -0.03 & 199.37 & 199.35 & 0.02 \\
\hline 27 & 138.64 & 138.64 & 0.00 & 140.38 & 140.39 & -0.01 \\
\hline 28,32 & 129.71 & 129.71 & 0.00 & 129.69 & 129.70 & -0.01 \\
\hline 29,31 & 129.18 & 129.19 & -0.01 & 129.48 & 129.47 & 0.01 \\
\hline 30 & 133.96 & 133.97 & -0.01 & 133.78 & 133.77 & 0.01 \\
\hline $2-\mathrm{OMe}$ & & & & 52.54 & 52.54 & 0.00 \\
\hline
\end{tabular}

